\title{
A new decision support framework for managing foot-and-mouth disease epidemics
}

\author{
Lan Ge • Anders R. Kristensen • Monique C. Mourits • \\ Ruud B. Huirne
}

Published online: 17 July 2010

(C) The Author(s) 2010. This article is published with open access at Springerlink.com

\begin{abstract}
Animal disease epidemics such as the foot-and-mouth disease (FMD) pose recurrent threat to countries with intensive livestock production. Efficient FMD control is crucial in limiting the damage of FMD epidemics and securing food production. Decision making in FMD control involves a hierarchy of decisions made at strategic, tactical, and operational levels. These decisions are interdependent and have to be made under uncertainty about future development of the epidemic. Addressing this decision problem, this paper presents a new decision-support framework based on multi-level hierarchic Markov processes (MLHMP). The MLHMP model simultaneously optimizes decisions at strategic, tactical, and operational levels, using Bayesian forecasting methods to model uncertainty and learning about the epidemic. As illustrated by the example, the framework is especially useful in contingency planning for future FMD epidemics.
\end{abstract}

Keywords Foot-and-mouth disease (FMD) - Epidemic control · Decision support · Bayesian forecasting · Multi-level hierarchic Markov processes (MLHMP)

L. Ge (凶)

LEI Wageningen UR, Hollandseweg 1, 6706KN, Wageningen, The Netherlands e-mail: Lan.Ge@wur.nl

\author{
A.R. Kristensen \\ Department of Large Animal Sciences, University of Copenhagen, Grønnegårdsvej 2, \\ 1870 Frederiksberg C, Denmark
}

M.C. Mourits

Business Economics Group, Wageningen University, Hollandseweg 1, 6706KN, Wageningen, The Netherlands

R.B. Huirne

Social Sciences Group, Wageningen UR, Hollandseweg 1, 6706KN, Wageningen, The Netherlands 


\section{Introduction}

For centuries, foot-and-mouth disease (FMD) has been a "curse" haunting the livestock industry around the world (Dijkhuizen et al. 1995; Yang et al. 1999; Randolph et al. 2002). FMD is a highly contagious viral disease affecting cattle, swine, and other cloven-hoofed animals. Although the mortality rate is in general low, the disease causes reduced milk yield and loss of weight. Outbreaks of FMD lead to substantial losses due to the costs of disease control, productivity losses and constraints on international meat and livestock trade (Paarberg and Lee 1998; Mahul and Durand 2000). The 2001 FMD epidemics in Europe exhibited their devastating impact on the economy and the society. In the United Kingdom the losses to agriculture and government amounted to about 4.5 billion Euro (Thompson et al. 2002). In the Netherlands, the economic damage was estimated to be around 1 billion Euro (Huirne et al. 2002). In the aftermath of these outbreaks, the decisions on control strategies raised heated debates (Anderson 2002; Cuijpers and Osinga 2002). The importance of decision making in managing FMD epidemics has attracted keen attention both in practice and in research.

A characteristic feature of decision making in FMD control is the uncertainty about current disease status and future epidemic development. Since the disease can only be detected (by clinical symptoms or serological tests) after the infection has taken place for sometime (incubation period), some infected animals can remain infectious without being detected, causing further spread of the disease. The number of these undetected but infectious herds is typically unknown to the decision maker at the time when FMD is detected. Even when these infectious herds were known, future spread of the disease is still uncertain due to variation in animals and randomness in the disease spread process (Keeling and Grenfell 2000).

Uncertainty about FMD spread imposes severe difficulty on the choice of control measures. In the EU, governments of member states are obliged to control FMD according to relevant regulations and international trade agreements (European Union 2003). The EU directives, while mandating a minimal set of control measures, leave it to the government of member states to decide whether additional measures should be taken to enhance the effectiveness of control. Additional measures such as pre-emptive culling (PC) or emergency vaccination $(\mathrm{EV})$ incur considerable upfront costs. Ideally, these measures should only be taken when their estimated benefits (resulting from the increased effectiveness of control) should at least offset their costs. Unfortunately, while the upfront costs are quite certain and irreversible, the benefits estimated ex ante are quite uncertain, which implies that the benefits ex post can significantly differ from the benefit estimated ex ante. Decisions on the additional measures must therefore deal with these uncertainties and the irreversibility of control measures (Mahul and Durand 2000).

Since an epidemic develops over time, decision making in FMD control is a sequential process in which control measures are adapted according to real-time development of the epidemic, i.e., a series of decisions will be made during the epidemic. Choice of additional control measures is therefore seldom a once-for-all or now-or-never decision, but must be dynamically considered and take into account the impact of early decisions on later decisions and the possibility of learning during the epidemic (Ge et al. 2005). The flexible timing of additional measures generates an infinite number of possible control strategies, bringing formidable complexities into decision making.

From a decision making point of view, managing FMD entails a hierarchy of interdependent decisions at three levels: strategic decisions, tactical decisions, and operational decisions (DEFRA 2004). Given the uncertainties and complexities, lessons learned from previous epidemics highlighted the strong need for a decision support framework which can 
be used to integrate information and knowledge about FMD control and guide further research (Anderson 2002). The objective of this study is to present such a decision support framework.

The framework is built upon the framework of multi-level hierarchic Markov processes (MLHMP) in which decisions are addressed at multiple timescales and simultaneously optimized (Kristensen and Jørgensen 2000). MLHMP inherits the stochastic and sequential nature of ordinary Markov decision processes (MDP) but circumvents the dimensionality problem by exploiting the hierarchic structure of the state space and decisions. When uncertainty exists about the state variables (e.g., when they are unobservable or partially observable), the sequential approach of MLHMP makes it possible to apply Bayesian updating between decision epochs to model learning effects (Kristensen 1993). Primarily developed as a decision support tool for herd management, MLHMP has been applied to decision problems within a herd concerning (endemic) infectious diseases, where disease control decisions must be made simultaneously with production and delivery decisions (Toft et al. 2005).

In this paper, we extend the MLHMP framework to epidemic control decisions that are made beyond herd or farm level, with governments as the decision maker. Bayesian updating techniques are applied by embedding a Poisson process with unknown parameter into the MLHMP framework. This extends the framework as presented by Nielsen et al. (2010) which embedded a linear state space model into a MLHMP.

We present the framework by first linking FMD control decisions to the decision processes of MLHMP at each level, then introducing the disease spread model, and subsequently illustrating how to embed decisions and the disease spread model into the MLHMP model. The MLHMP model provides a scientific representation of the decision problem and calculates the optimal control strategy. A simple example is developed while introducing the framework. Further empirical applications of the framework are then discussed.

\section{The decision hierarchy}

\subsection{Modelling FMD control decisions with MLHMP}

The MLHMP technique exploits the hierarchic structure of decisions and state variables in managing a dynamic system. Technically, the essence of the MLHMP technique is to build ordinary MDPs with shorter time horizon (called child processes) into MDPs with longer time horizon (called parent processes). The parent process with the longest time horizon is called the founder process, which is the main decision process in MLHMP. The hierarchy of these decision processes corresponds naturally to decisions of strategic, tactical, and operational nature in FMD control. As an example, a 3-level MLHMP structure is illustrated in Fig. 1.

At the founder level (strategic decisions) we show one decision stage of the founder process. The founder process can have finite or infinite time horizon. Like ordinary MDPs, the process has states, actions and decision epochs. Unlike ordinary MDPs, an action taken in a founder state does not cause a direct transition to a founder state in the next decision epoch. Instead, it initiates a decision process at Child 1 level (tactical decisions), which is a finite horizon MDP with $T_{1}$ stages. Similarly, an action taken in a Child 1 state initiates a decision process at Child 2 level (operational decisions), which is a finite horizon MDP with $T_{2}$ stages. At the lowest level (i.e. Child 2), an action does cause regular state transitions. The use of MDPs captures the sequential and stochastic nature of the decision process at 


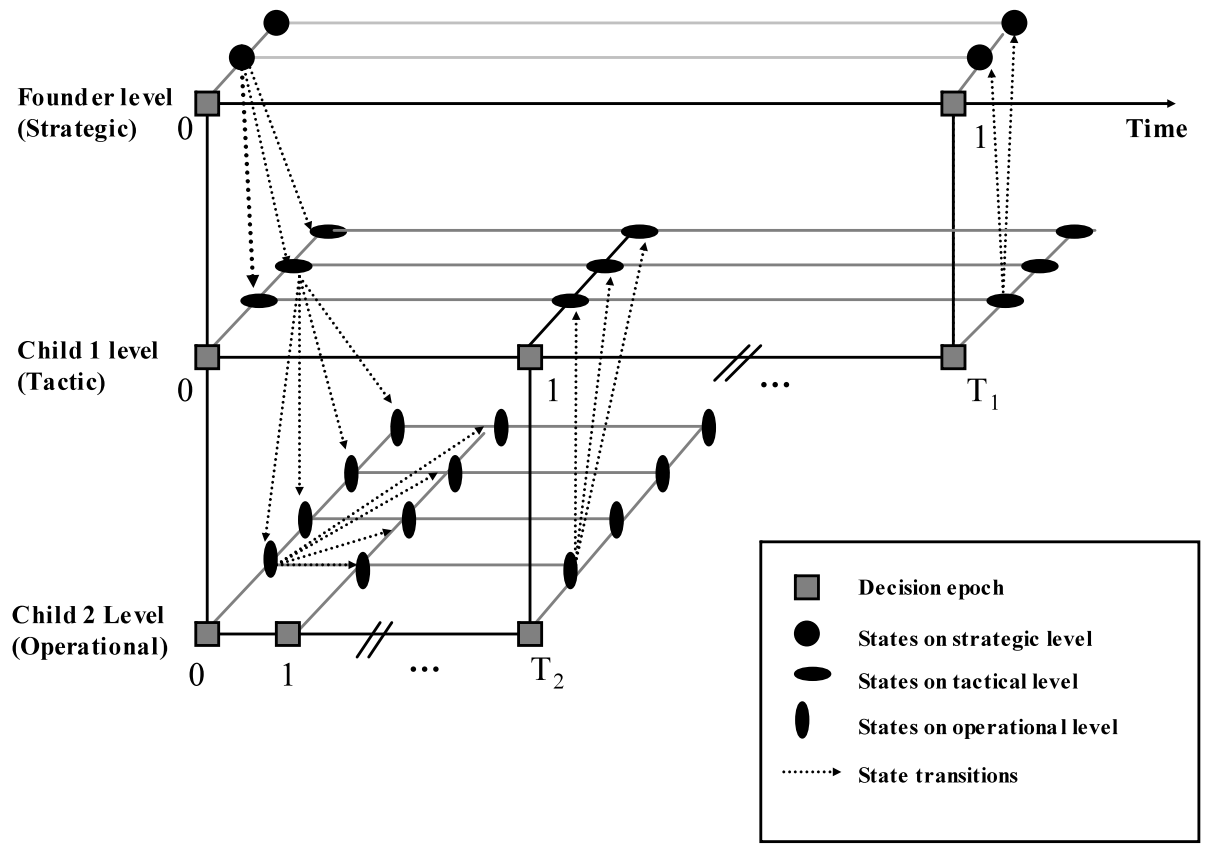

Fig. 1 The MLHMP representation of the hierarchic decision space

each level. The art of using MLHMP is to define decisions at the proper levels in terms of their time horizons, states, decision stages, actions, rewards and state transitions. For FMD control, the decisions are modelled as follows.

\subsection{Strategic decisions}

Strategic decisions in FMD control take the form of veterinary policies, which have longterm impact and address states of the system that can change on the long term, but remain unchanged during one epidemic. Typically, strategic decisions are made before an epidemic occurs and determine the range of control options. One example of strategic decisions is whether preventive vaccination should be used. Having made a strategic decision, the tactical decisions are restricted. For example, if the strategic decision is "no vaccination should be used", there would be no need to consider a vaccination program during an epidemic.

Actions for strategic decisions are often confined by relevant legal obligations from the national and communal government. For example, the EU had decided in 1991 to stop routine vaccination against FMD, relying on efficient control program to recover FMD-free status in the case of FMD epidemic (Horst et al. 1999). The Council Directive 2003/85/EC (of September 29, 2003), however, allows emergency vaccination as an additional control measure in case of an FMD epidemic (European Union 2003).

\subsection{Tactical decisions}

If strategic decisions determine the range of control options for all epidemics, tactical decisions concern the deployment of control options for one specific epidemic. The time horizon of tactical decisions is shorter than one epidemic. Depending on the actual duration of the 
epidemic, one epidemic may involve a sequence of tactical decisions over time. The choice of the time horizon of tactical decisions depends on the length of incubation period and logistic capacity.

A tactical decision at the start of an epidemic can be, for example, whether or not to implement additional control measures like pre-emptive culling (PC) or emergency vaccination (EV), or to use the compulsory measures only (i.e., basic program (BP)), and decide at the next decision stage whether or not to implement PC or EV. For a particular epidemic, tactical decisions are the most important decisions to be made, since the strategic decisions were made prior to the epidemic and the operational decisions must follow tactical decisions.

\subsection{Operational decisions}

Operational decisions in FMD control concern the execution of the chosen tactical decisions. Time horizon of the operational decisions is the shortest in the decision hierarchy. Conditional on the tactical decision, actions considered by an operational decision is typically whether or not to continue the tactical choice. Due to the short stage length of operational decisions, uncertainties at operational level are lower than those at tactical and strategic level. Since the epidemic states can be observed daily, short term prediction on the epidemic status can be immediately checked and updated. Learning is therefore an important feature to be modelled at the operational level.

\section{Modelling FMD spread: dynamic models}

The "curse" of FMD is to a large extent ascribable to the invisible spread of the virus which brings uncertainty about epidemic development. In understanding and modelling an FMD epidemic, a clear distinction should be made between two closely related processes, i.e. the spread of infection, which can not be completely observed, and the spread of disease, which is observed/detected (Green and Medley 2002).

As shown in many epidemiological models, assuming a stochastic process for the infection process, the observed spread of disease can be used to estimate the parameters for the stochastic process (see, e.g. Meester et al. 2002; Streftaris and Gibson 2004). Since the latent process is evolving, the parameters for the stochastic process will also evolve over time, which means dynamic models are appropriate to model FMD spread (West and Harrison 1997).

\subsection{Observation model and forecasting}

Assume the number of new FMD detections (herds) at time $t, N_{t}$, is drawn from a Poisson distribution with parameter $\lambda_{t}$, i.e.

$$
p\left(N_{t} \mid \lambda_{t}\right)=\frac{e^{-\lambda_{t}} \lambda_{t}^{N_{t}}}{N_{t} !}
$$

where $p\left(N_{t} \mid\right.$.) is the conditional probability of $N_{t}$, and $\lambda_{t}>0$ is the expectation and variance of $N_{t}$ at time $t$.

The parameter for the Poisson distribution, $\lambda_{t}$, determines the irreducible variability of the observations at time $t$. In other words, even if this parameter is perfectly known, the actual observation is still random at time $t$. If $\lambda_{t}$ has to be estimated, the subjective uncertainty would increase the total variability of the predicted outcome (for a good discussion 
on this issue, see Vose 2000). This subjective uncertainty can be represented as a probability distribution of $\lambda_{t}$. Since the Gamma distribution is often used as a conjugate prior for Poisson distribution in Bayesian forecasting (DeGroot 1970), we therefore assume that prior to the observation of $N_{t}, \lambda_{t}$ is drawn from a Gamma distribution with parameters $\alpha_{t}$ and $\beta_{t}$, i.e., $\left(\lambda_{t} \mid D_{t-1}\right) \sim \operatorname{Gamma}\left(\alpha_{t}, \beta_{t}\right)$, where $D_{t-1}$ is the information set before observing $N_{t}$, i.e. $D_{t-1}=\left\{N_{1}, \ldots, N_{t-1}\right\}$. The predicted number of detection at time $t, N_{t}$, will then follow a Gamma-Poisson distribution. Based on this distribution, the one-step forecast distribution of $N_{t}, p\left(N_{t} \mid D_{t-1}\right)$, can be derived from the density functions of a Gamma distribution and a Poisson distribution as follows:

$$
p\left(N_{t} \mid D_{t-1}\right)=\frac{\beta_{t}^{\alpha_{t}}}{\left(\beta_{t}+1\right)^{\left(\alpha_{t}+N_{t}\right)} N_{t} !} \prod_{i=1}^{N_{t}}\left(\alpha_{t}+N_{t}-i\right) .
$$

This forecast distribution is used to derive state transition probabilities, which will be explained in Sect. 4.1.

\subsection{Epidemic progression and learning}

At any time $t$, the estimation on $\lambda_{t}$ is based of the information set $D_{t}=\left\{N_{1}, \ldots, N_{t}\right\}$. To efficiently summarize the information on past observations, we use the exponentially weighted moving average (EWMA) of previous numbers of detections as a proxy for the observed level of epidemic (Montgomery 2005). The EWMA is the statistic

$$
J_{t}=\gamma N_{t}+(1-\gamma) J_{t-1}
$$

where $\gamma(0 \prec \gamma \prec 1)$ is a weighting factor showing the relative importance of historical data to current data in forecasting. In practice, the appropriate $\gamma$ is chosen to minimize the forecast error. Denote this statistic as infection index $\left(J_{t}\right)$, it would be reasonable to assume that the number of detections at time $t$ depends on the infection index at time $t-1$. Suppose the relationship can be described as:

$$
E\left(N_{t} \mid D_{t-1}\right)=\lambda_{t}=J_{t-1} \theta_{t}
$$

Further assume that the effect of control action $a$ at time $t$ can be estimated as a reduction factor $\delta_{t}^{a}\left(0 \leq \delta_{t}^{a} \leq 1\right)$ on the latent variable $\theta_{t}$ and as a result:

$$
E\left(N_{t}\right)=\lambda_{t}=F_{t} \theta_{t}=\delta_{t}^{a} J_{t-1} \theta_{t}
$$

The latent variable $\theta_{t}$ can be interpreted as a growth potential of the epidemic, which is a property of the virus. If the property of the FMD virus does not change during the epidemic, the evolution of $\theta_{t}$ can be described with a constant model, i.e.:

$$
\theta_{t}=\theta_{t-1}
$$

An important consideration to use dynamic models is that they provide a sound mechanism to model uncertainty and learning through Bayesian updating. Suppose at time $t$, uncertainty of the unobservable variable $\theta_{t}$ before observing $N_{t}$ can be described as a partly specified distribution $\left[m_{t}, C_{t}\right]$, where $m_{t}$ and $C_{t}$ are the first and second moment. This distribution is the prior distribution for $\theta_{t}$. After observing $N_{t}$, the posterior distribution for $\theta_{t}$ can be calculated according to the updating procedure for this type of dynamic generalized linear models (DGLM) described in West and Harrison (1997). 
Table 1 Information about decisions and states in the example MLHMP model

\begin{tabular}{|c|c|c|c|c|c|}
\hline $\begin{array}{l}\text { MLHMP } \\
\text { level }\end{array}$ & $\begin{array}{l}\text { Decision } \\
\text { level }\end{array}$ & $\begin{array}{l}\text { Time } \\
\text { horizon }\end{array}$ & Stage length & State variable(s) & Level-specific action(s) \\
\hline Founder & Strategic & Infinite & $\begin{array}{l}\text { Maximal length } \\
\text { of an epidemic } \\
\left(=T_{1} \times T_{2} \text { days }\right)\end{array}$ & $\begin{array}{l}\text { Epidemic situation } \\
\text { ( } 2 \text { levels, i.e., with or } \\
\text { without epidemic) }\end{array}$ & $\begin{array}{l}\text { 1. No control action } \\
\text { 2. Start control programs }\end{array}$ \\
\hline \multirow[t]{3}{*}{ Child 1} & \multirow[t]{3}{*}{ Tactical } & \multirow{3}{*}{$\begin{array}{l}T_{1} \text { periods } \\
\left(T_{1}=10\right)\end{array}$} & \multirow{3}{*}{$\begin{array}{l}\text { One period } \\
\left(=T_{2} \text { days }\right)\end{array}$} & 1. $J_{t}$ (5 levels ) & 1. Basic program only (BP) \\
\hline & & & & 2. $m_{t}$ (5 levels) & $\begin{array}{l}\text { 2. Basic program }+ \\
\text { Emergency vaccination } \\
(\mathrm{EV})\end{array}$ \\
\hline & & & & 3. $C_{t}$ (5 levels) & $\begin{array}{l}\text { 3. Basic program }+ \\
\text { Pre-emptive culling }(\mathrm{PC})\end{array}$ \\
\hline \multirow[t]{3}{*}{ Child 2} & \multirow[t]{3}{*}{ Operational } & \multirow[t]{3}{*}{$\begin{array}{l}T_{2} \text { days } \\
\left(T_{2}=10\right)\end{array}$} & \multirow[t]{3}{*}{1 day } & \multirow[t]{3}{*}{$\begin{array}{l}\text { Same as Child } 1 \\
\text { level }\end{array}$} & $\begin{array}{l}\text { 1. Start or continue the } \\
\text { program chosen (Start) }\end{array}$ \\
\hline & & & & & $\begin{array}{l}\text { 2. Stop the chosen } \\
\text { program (Stop) }\end{array}$ \\
\hline & & & & & $\begin{array}{l}\text { 3. Stop all control } \\
\text { programs (End) }\end{array}$ \\
\hline
\end{tabular}

\section{The MLHMP model}

The FMD spread model represents the key knowledge and uncertainty of the decision-maker about the disease and the effects of control measures. Such a model is needed to define state variables and derive state transition probabilities for the MLHMP model. The choice of states, actions and rewards depends on the specific questions to be answered by the model. Accordingly, the MLHMP model can be specified in different ways. In this session we describe the specification of the MLHMP-parameters for the relevant decisions and illustrate it with the example. Information about the state actions and state length of the MLHMP example is listed in Table 1.

\subsection{States and state transitions}

The choice of state variables and states depends on the strategic decisions to be considered. At the strategic level, overall disease status is an obvious state variable of which states can be defined as: FMD-free, FMD epidemic, and FMD-endemic. Social-economic variables can be used as state variables as well due to their impact on the social-economic consequences of FMD control. To simplify the illustration, we considered only one state variable at the strategic level with two states: FMD epidemic and FMD-free. Possible actions at the strategic level are start control programs and take no control action.

Practice shows that decision support is mostly needed for tactical decisions since strategic decisions are to a great extent confined by legal framework and have less states and actions. At tactical and operational levels (Child 1 and Child 2), decisions involve more states and actions and the actions need to be fine-tuned to the states. For the example, a state at decision stage $t$ is defined by the value of the following three state variables: the current infection index $J_{t}$; the posterior mean, $m_{t}$, of the underlying parameter $\theta_{t}$; and the posterior variance, $C_{t}$, of the underlying parameter $\theta_{t}$. We denote $i=\left(J_{i t}, m_{i t}, C_{i t}\right)$ for state $i$ when convenient. A state describes therefore both observed epidemic status $\left(J_{t}\right)$, expected epidemic growth $\left(m_{t}\right)$, and the uncertainty about the growth $\left(C_{t}\right)$. 
At the founder level of an MLHMP model, state transition probabilities are often specified. At other parent levels, the transition probabilities are calculated from corresponding child processes as described by Kristensen and Jørgensen (2000). At the lowest child level (i.e., Child 2), transition probabilities are calculated from the epidemic spread model. Denote $p_{i j}^{a}(t)$ as the transition probability from state $i=\left(J_{i t}, m_{i t}, C_{i t}\right)$ at stage $t$ to state $j=\left(J_{j, t+1}, m_{j, t+1}, C_{j, t+1}\right)$ at stage $t+1$. For given $J_{i t}$ and $J_{j, t+1}$, let $M\left(J_{i t,} J_{j, t+1}\right)$ denote the set of values of $N_{t+1}$ resulting in updating of $J_{i t}$ to $J_{j, t+1}$. If $M\left(J_{i t,} J_{j, t+1}\right)=\varnothing$ (empty set), $p_{i j}^{a}(t)=0$. Otherwise, $p_{i j}^{a}(t)$ is calculated as follows:

$$
p_{i j}^{a}(t)=\sum_{k \in M\left(J_{i t}, J_{j, t+1}\right)} \frac{\beta_{t+1}^{\alpha_{t+1}}}{\left(\beta_{t+1}+1\right)^{\left(\alpha_{t+1}+k\right)} k !} \prod_{n=1}^{k}\left(\alpha_{t+1}+k-n\right) \quad(\text { cf. (2)) }
$$

if $m_{j, t+1}$ and $C_{j, t+1}$ conform the calculated posterior distribution of $\theta_{t+1}$, otherwise $p_{i j}^{a}(t)=0$.

The three state variables $J_{t}, M_{t}$, and $C_{t}$ are continuous variables. To derive finite states needed for the MLHMP model they need to be discretized into intervals. The appropriate number and range of the intervals should be chosen based on the desired precision of the optimal strategy and the sensitivity of the optimal strategy to the state variables. The more sensitive the optimal strategy is to the state variables, the more intervals the state variable should be discretized. For simplicity, we have discretized all three state variables into 5 levels only.

\subsection{Actions and rewards}

The actions at different decision levels are shown in Table 1. The rewards (costs) of actions must be defined according to the objective(s) of the decision maker. It is worth noting that different objectives can lead to different "optimal" strategies. To provide decision support, it is important to clearly state the decision objective and let the objective guide the selection of state variables.

For the example, it is supposed that the objective of the government is to minimize the total direct control costs. In this case, the reward is in the form of costs (or negative rewards). Direct costs in this case are labour and material costs incurred by the control programs as well as compensations paid to the affected parties. Other objective can be to minimize the sum of direct control costs and export losses to the livestock section (Tomassen et al. 2002). Since the pre-emptive culling of healthy animals can cause psychological damage to the society, minimizing the number of healthy animals slaughtered can be an objective as well.

In general, direct costs of FMD control depend on the number of herds detected. Denote $U_{a}\left(N_{t}\right)$ as the reward (cost) function for action $a$ when the number of new detection is $N_{t}$. The form and parameters for the function $U_{a}$ (.) can usually be specified based on economic analyses. The expected reward for state $i$ of Child 2 level if action $a$ is taken at stage $t, r_{i}^{a}(t)$, is then:

$$
r_{i}^{a}(t)=E\left[U_{a}\left(N_{t+1}\right) \mid D_{t}, i, a\right]=\sum_{N_{t+1}=0}^{\infty} U_{a}\left(N_{t+1}\right) P\left(N_{t+1} \mid D_{t}, i, a\right) .
$$

Since the probability is calculated as (2), this becomes:

$$
r_{i}^{a}(t)=\sum_{N_{t+1}=0}^{\infty} U_{a}\left(N_{t+1}\right) \frac{\beta_{t+1}^{\alpha_{t+1}}}{\left(\beta_{t+1}+1\right)^{\left(\alpha_{t+1}+N_{t+1}\right)} N_{t+1} !} \prod_{n=1}^{N_{t+1}}\left(\alpha_{t+1}+N_{t+1}-n\right) .
$$


Table 2 Parameters and values used in the example MLHMP model

\begin{tabular}{lll}
\hline Parameter & Interpretation & Example value \\
\hline$\gamma$ & Weighting factor for the infection index & 0.11 \\
$\delta_{1}$ & The reduction ratio for basic program & 0.40 \\
$\delta_{2}$ & The reduction ratio for preemptive culling & 0.20 \\
$\delta_{3}$ & The reduction ratio for emergency vaccination & 0.30 \\
$J_{\max }$ & Lower bound for the highest level of $J_{t}$ & 0.15 \\
$J_{\min }$ & Upper bound for the lowest level of $J_{t}$ & 3.0 \\
$m_{\max }$ & Lower bound for the highest level of $m_{t}$ & 5.0 \\
$m_{\min }$ & Upper bound for the lowest level of $m_{t}$ & 1.0 \\
$C_{\max }$ & Lower bound for the highest level of $C_{t}$ & 1.5 \\
$C_{\min }$ & Upper bound for the lowest level of $C_{t}$ & 0.2 \\
$C_{0}$ & Basic epidemic costs per day during the epidemic & $4,300 \mathrm{k} €$ \\
$C_{B P}$ & Additional costs of basic program per newly detected herd & $100 \mathrm{k} €$ \\
$C_{P C}$ & Additional costs of preemptive culling per newly detected herd & $6,500 \mathrm{k} €$ \\
$C_{E V}$ & Additional costs of emergency vaccination per newly detected herd & $400 \mathrm{k} €$ \\
\hline
\end{tabular}

The rewards at the founder level are calculated from the child levels as described in Kristensen and Jørgensen (2000). To simplify the illustration, we specify $U_{a}\left(N_{t}\right)=C_{0}+C_{a} N_{t}$, where $C_{0}$ are the fixed costs of epidemic control (for all control programs), and $C_{a}$ is the additional costs per detected cases should program $a$ be implemented. This specification gives $r_{i}^{a}(t)=E\left[U_{a}\left(N_{t+1}\right)\right]=U_{a}\left[E\left(N_{t+1}\right)\right]$, which simplifies the calculation of $r_{i}^{a}(t)$ since $E\left(N_{t+1}\right)$ can be derived directly from (5). The parameters used for the example are listed in Table 2 .

\subsection{Results}

Optimization of a MLHMP model generates optimal control strategy for the chosen optimality criterion. The optimal control strategy consists of decision rules for all possible states which assign the optimal action to each state. The decisions at the strategic level are straightforward: start control when there is FMD epidemic and no control action is taken otherwise. At operational level, the decision to stop the control program is assigned to the state with lowest level of $J_{t}$ (i.e., the epidemic is considered to be over), otherwise the tactical choice will be carried out.

As mentioned in Sect. 2, tactical decisions are most important for the control of a particular epidemic which need to be fine-tuned to the states. A fraction of the optimal tactical decisions for the example is shown in Table 3. Table 3 shows that initial choice of the tactical decision (Period 1) varies in different states. For many states, it is optimal to start with the basic program (BP) only. Choices of control programs at later stages depend on to which state the current state will make a transition. For example, the initial choice for Child 1 state No. 27 is to use BP. In Period 2, if the epidemic goes to state No. 6, emergency vaccination (EV) should be used. And if the epidemic goes to state No. 39, pre-emptive culling (PC) should be used.

Since the state transitions are probabilistic, it is not certain in Period 1 which state the epidemic will enter in Period 2. However, for each possible state, the optimal action to be taken is known in Period 1. The same holds for decisions at later stages. The results of the 


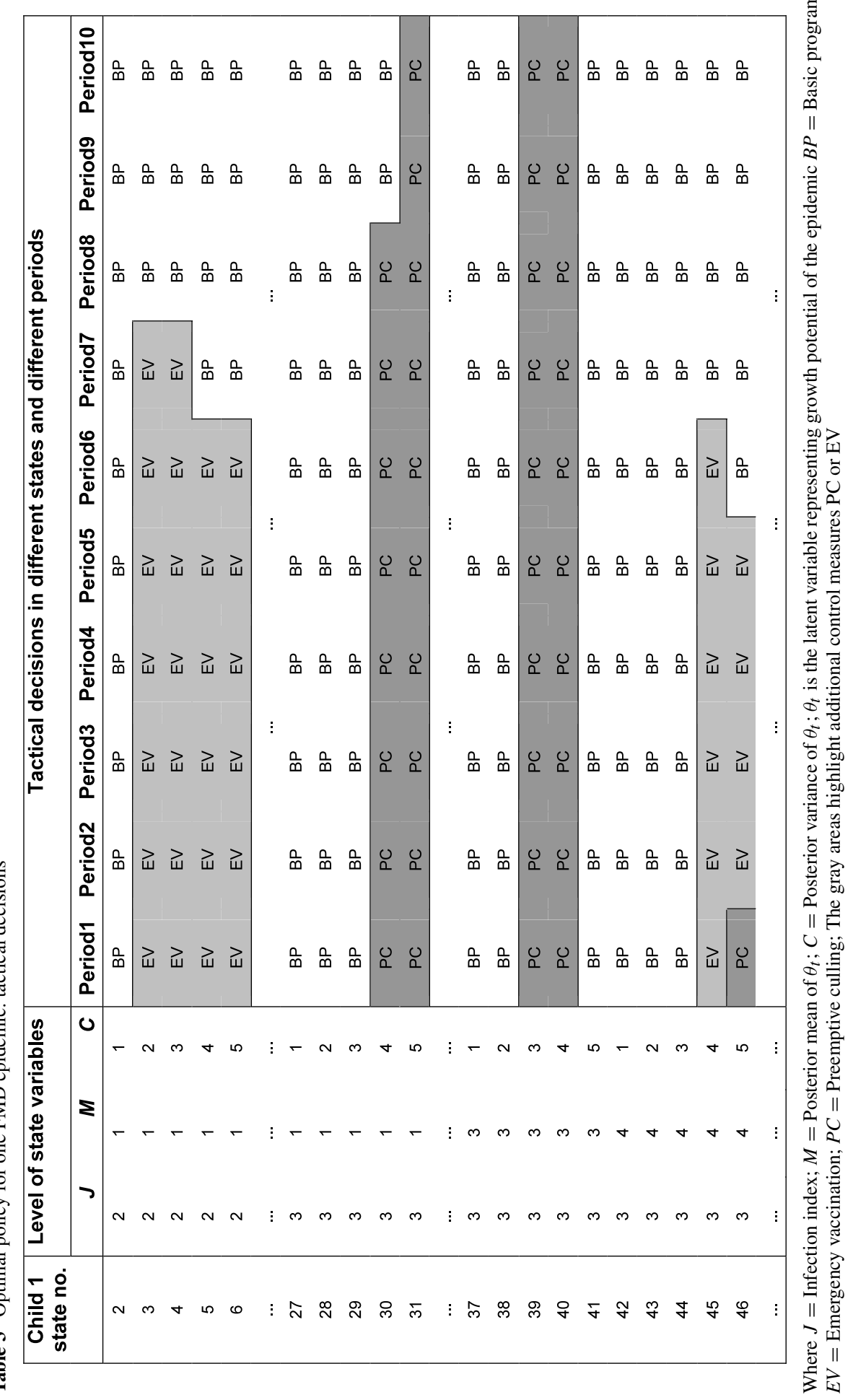


MLHMP model constitute a contingency plan for all possible scenarios of the epidemic (as described by the states). The contingency plan provides guideline for FMD control which ensures that the expected costs of FMD control is minimized if the strategy is followed.

The optimization of the MLHMP model calculates in fact expected costs of all possible strategies to find the optimal strategy. This means that expected costs of both the optimal strategy and the sub-optimal strategies (optimal action is not used) are available. Difference in these results indicates the risk of using sub-optimal control strategies and the robustness of the optimal strategy. For example, using the costs given in Table 2 we can calculate that using the suboptimal action PC in state No. 27 (Period 1) instead of BP would increase the total costs by about 66 million $€$. This means that postponing using additional control measures such as PC in the first period can avoid unnecessary control costs.

\section{Discussion}

The decision support framework presented above provides a sound theoretical basis to deal with uncertainties, learning, and hierarchic decisions in the dynamic decision making of FMD control. The example demonstrates the potential of the framework for empirical application. In particular, the results of the MLHMP model constitute a contingency plan which can be used to be prepared for future epidemics.

Empirical application of the framework means obtaining realistic MLHMP parameters (decision parameters, epidemiological parameters, and economic parameters) and using the MLHMP model for contingency planning. This section addresses these issues.

\subsection{Model parameters}

Most information listed in Table 1 can be determined by consulting relevant policy documents such as the contingency plans. The number of levels for each state variable should be chosen to balance the practical need and computational convenience. Since the state variables are defined on a continuous scale, discretization will always violate the embedded state space model to a certain extent. To approximate the continuous scale of the state variables, more levels are in general preferred to less. However, more levels will increase the state space explosively and might lead to computational infeasibility. Sensitivity analysis may be carried out to select the appropriate number of levels based on their impact on the optimal policy.

Parameters such as listed in Table 2 need additional epidemic and economic modelling, using information from past epidemics, experimental data and simulated epidemics (see e.g., Ge et al. 2010). A large variety of economic and epidemiological models and methods can be used for this purpose. For example, realistic epidemics in the Netherlands can be simulated with the spatial stochastic simulation model InterFMD, using farm census data (see e.g. Velthuis and Mourits 2007).

Considering the importance of the parameters of the disease spread model when defining states and calculate state transition probabilities, methods to estimate these parameters are worth describing. The weighing factor $\gamma$ and initial moments for $\theta_{0}$ i.e. $\left\{m_{0}, C_{0}\right\}$, can be estimated as follows.

Step 1. Start the infection and detection process in the simulation model with one farm (randomly drawn from all susceptible herds) as the index case (first infected case). 
Step 2. When the first case is detected, the time is recorded as time 1 of the epidemic. Note the number of detected herd on this day as $N_{1}$ (i.e. $N_{1}=1$ ) and the number of infected but undetected herds as $I_{0}$. Also note the number of detected herds after this day as $N_{2}, \ldots, N_{T}$, where $T$ is the maximal duration of an epidemic.

Step 3. Repeat Step 2 for $M$ times to get $M$ values for $I_{0}$ and $M$ time series of $\left\{N_{1}, \ldots, N_{T}\right\}$. Step 4. Calculate the mean $\left(m_{0}\right)$ and variance $\left(C_{0}\right)$ of $I_{0}$ from the $M$ time series. Since the undetected but infected herds $I_{0}$ determine the potential growth of the observed epidemic, it can be seen as $\theta_{0}$ in (5) (with $J_{0}=1$ and $\delta=1$ ). The estimates $m_{0}$ and $C_{0}$ can therefore be used to calculate $\alpha_{0}$ and $\beta_{0}$ as described in West and Harrison (1997).

Step 5. For each time series, repeat the following procedure for multiple $\gamma$ values, $\gamma=$ $\{0.1,0.2, \ldots, 0.9\}$ :

- Run the DGLM for each time series

- On each day, use (5) (with $\delta=1$ ) as a predictor for $N_{t+1}$

- Calculate the forecast error $e_{t}=N_{t+1}-f_{t}$, where $f_{t}$ is the predictor

- Calculate the total sum of squares of the forecast errors for all $M$ series.

Step 6. Choose the $\gamma$ minimizing the total sum of squares of the forecast errors.

The parameter $\delta_{1}, \delta_{2}$, and $\delta_{3}$ can be estimated similarly by simulating the epidemic with the actions implemented. It should be noted that, as an optimization model, the MLHMP framework uses highly aggregated data and parameters and ignores the detailed information that is available when simulation models are used. For example, the spatial information contained in the GIS data could be used to provide region-specific or even farm-special control measures. Even though the information loss caused by data aggregation is inevitable, the impact should be investigated with sensitivity analyses by altering the level of aggregation. Further methodological research is necessary to investigate how to include spatial information in the MLHMP model.

\subsection{Contingency planning}

Even though FMD is a constant threat, the time and situation in which the next FMD outbreak will occur is unpredictable. To increase preparedness, it is important to carry out contingency planning during "peace time". Empirical application of the MLHMP framework can contribute to efficient contingency planning in two ways. First, it can be used to compare optimal strategies under different decision objectives by changing reward functions and the optimization criteria. If multiple objectives exist, investigating optimal strategies under different objectives can facilitate the weighing process in multi-criteria analysis.

The second application of the framework makes use of the feature that the solution of a MLHMP model generates not only the optimal strategy, but also calculates results of alternative strategies. These results can be used to investigate the robustness of the optimal strategy. One way to gain insight into the robustness of the optimal strategy in particular state is to compare the results of the optimal strategy with a suboptimal strategy which uses alternative action in the same state, as was done with the example. Based on the Markov chain and value functions generated by the optimization, the results from other suboptimal strategies can be investigated by replacing more actions from the optimal strategy and simulate the results with the underlying Markov chain. It is worth mentioning that, given an optimal policy, the variance of the policy can be calculated from the simulations. This can facilitate the risk communication with respect to the possible consequences of epidemic control. 
The theory of MLHMP and the applications described above may remain abstract for more practical application. To make the method more accessible, a software system has been developed by Kristensen (2003). This software, visualizing the structure of the decision model, provides a graphical interface between real-life decision problem and the corresponding decision model. This feature can facilitate the communication of the contingency plan among different stakeholders.

\section{Concluding remarks}

This paper presents a decision support framework which deals with the uncertainties and complexities of decision making in controlling FMD epidemics. The decision problem in FMD control is represented by an MLHMP model which, when calculating the optimal control strategy, takes into account the interdependency among decisions. The use of dynamic models and Bayesian updating provides consistent treatment to uncertainties and learning about epidemic development. By identifying the hierarchic structure of the decision complex, the framework simultaneously optimizes strategic, tactical, and operational decisions.

The decision support framework offers promising perspectives for empirical applications. As shown by the example, an important application of the framework is contingency planning for future epidemics. The MLHMP model not only generates the optimal strategy, but also provides information on suboptimal strategies, which can be used to test the robustness of the optimal strategy. Depending on the desired level of details, the models can be extended to answer specific questions of the decision-maker. The framework we have illustrated is of generic nature and can be extended to other highly contagious epidemic diseases such as classical swine fever (CSF) and avian influenza.

Open Access This article is distributed under the terms of the Creative Commons Attribution Noncommercial License which permits any noncommercial use, distribution, and reproduction in any medium, provided the original author(s) and source are credited.

\section{References}

Anderson, I. (2002). Foot and mouth disease 2001: lessons to be learned inquiry report. London: The Stationery Office.

Cuijpers, M. P., \& Osinga, K. J. (2002). The position of the Dutch Farmers' Union on lessons learned and future prevention and control of foot and mouth disease. Scientific and Technical Review of the OIE 21 (pp. 839-850).

DEFRA (2004). Foot and mouth disease contingency plan. Department for Environment, Food and Rural Affairs (DEFRA), UK.

DeGroot, M. H. (1970). Optimal statistical decisions. New York: McGraw-Hill.

Dijkhuizen, A. A., Huirne, R. B. M., \& Jalvingh, A. W. (1995). Economic analysis of animal diseases and their control. Preventive Veterinary Medicine, 25, 135-149.

European Union (2003). Council Directive 2003/85/EC of 29 September 2003 on Community measures for the control of foot-and-mouth disease repealing Directive 85/511/EEC and Decisions 89/531/EEC and 91/665/EEC and amending Directive 92/46/EEC. Official Journal of the European Union L306.

Ge, L., Mourits, M. C. M., \& Huirne, R. B. M. (2005). Valuing flexibility in the control of contagious animal diseases. In The 9th annual international conference on real options, Paris.

Ge, L., Mourits, M. C. M., Kristensen, A. R., \& Huirne, R. B. M. (2010). A modelling approach to support dynamic decision-making in the control of FMD epidemics. Preventive Veterinary Medicine, 95, 167174.

Green, L. E., \& Medley, G. F. (2002). Mathematical modelling of the foot and mouth disease epidemic of 2001: strengths and weaknesses. Research in Veterinary Science, 73, 201-205. 
Horst, H. S., de Vos, C. J., Tomassen, F., \& Stelwagen, J. (1999). The economic evaluation of control and eradication of epidemic livestock diseases. Scientific and Technical Review of the OIE, 18, 367-379.

Huirne, R. B. M., Mourits, M., Tomassen, F., de Vlieger, J. J., \& Vogelzang, T. A. (2002). FMD: past, present, and future. The Hague: LEI (in Dutch).

Keeling, M. J., \& Grenfell, B. T. (2000). Individual-based perspectives on $\mathrm{R}_{0}$. Journal of Theoretical Biology, 51-61.

Kristensen, A. R. (1993). Bayesian updating in hierarchic Markov processes applied to the animal replacement problem. European Review of Agricultural Economics, 20, 223-239.

Kristensen, A. R. (2003). A general software system for Markov decision processes in herd management application. Computers and Electronics in Agriculture, 38, 299-215.

Kristensen, A. R., \& Jørgensen, E. (2000). Multi-level hierarchic Markov processes as a framework for herd management support. Annals of Operation Research, 94, 69-89.

Mahul, O., \& Durand, B. (2000). Simulated economic consequences of foot-and-mouth disease epidemics and their public control in France. Preventive Veterinary Medicine, 47, 23-38.

Meester, R., de Koning, J., de Jong, M. C. M., \& Diekmann, O. (2002). Modeling and real-time prediction of classical swine fever epidemics. Biometrics, 58, 178-184.

Montgomery, D. C. (2005). Introduction to statistical quality control. New York: Wiley.

Nielsen, L. R., Jøgensen, E., \& Højsgaard, S. (2010). Embedding a state space model into a Markov decision process. Annals of Operations Research. doi:10.1007/s10479-010-0688-Z

Paarberg, P. L., \& Lee, J. G. (1998). Import restrictions in the presence of a health risk: an illustration using FMD. American Journal of Agricultural Economics 80, 175-183.

Randolph, T. F., Perry, B. D., Benigno, C. C., Santos, I. J., Agbayani, A. L., Coleman, P., Webb, R., \& Gleeson, L. J. (2002). The economic impact of foot and mouth disease control and eradication in the Philippines. Scientific and Technical Review of the OIE, 21, 645-661.

Streftaris, G., \& Gibson, G. J. (2004). Bayesian analysis of experimental epidemics of foot-and-mouth disease. Proceedings of the Royal Society of London B, Biological Sciences 271, 1111-1117.

Thompson, D., Muriel, P., Russell, D., Osborne, P., Bromley, A., Rowland, M., Creigh-Tyte, S., \& Brown, C. (2002). Economic costs of the foot and mouth disease outbreak in the United Kingdom in 2001. Scientific and Technical Review of the OIE, 21, 675-687.

Toft, N., Kristensen, A. R., \& Jorgensen, E. (2005). A framework for decision support related to infectious disease in slaughter pig fattening units. Agricultural Systems, 85, 120-137.

Tomassen, F. H. M., de Koeijer, A., Mourits, M. C. M., Dekker, A., Bouma, A., \& Huirne, R. B. M. (2002). A decision-tree to optimise control measures during the early stage of a foot-and-mouth disease epidemic. Preventive Veterinary Medicine, 54, 301-324.

Velthuis, A. G. J., \& Mourits, M. C. M. (2007). Effectiveness of movement-prevention regulations to reduce the spread of foot-and-mouth disease in Netherlands. Preventive Veterinary Medicine 82, 262-281.

Vose, D. (2000). Risk analysis: a quantitative guide. Chichester: Wiley.

West, M., \& Harrison, J. (1997). Bayesian forecasting and dynamic models. New York: Springer.

Yang, P. C., Chu, R. M., Chung, W. B., \& Sun, H. T. (1999). Epidemiological characteristics and financial costs of the 1997 foot-and-mouth disease epidemic in Taiwan. The Veterinary Record, 145, 731-734. 